FACULDADE DE CIÊNCIAS ECONô MICAS dA UFRGS
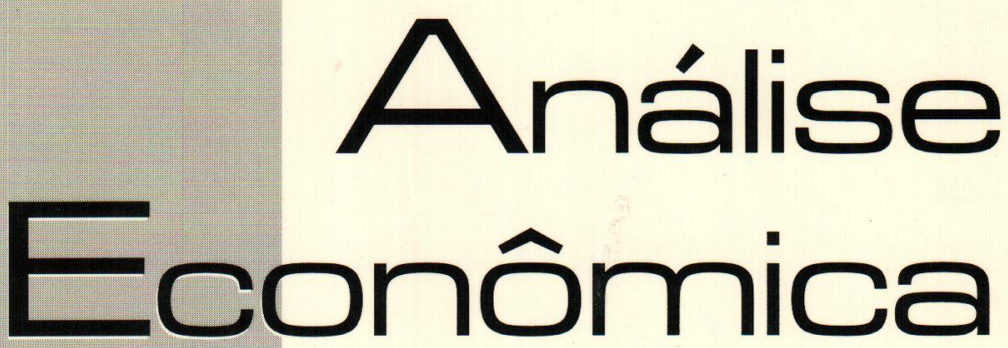

ESCOLHA DE PORTFÓLIO, INVESTIMENTO E NĀO. NEUTRALIDADE DA MOEDA

JOSÉ LUIS OREIRO

REFORMAS NA AROUITETURA FINANCEIRA INTERNACIONAL: NOVIDADES NO FRONT? ANDRÉ MOREIRA CUNHA

MERCOSUR'S CHANGE IN TRADE PATTERNS ANDRÉ FILIPE ZAGO DE AZEVEDO

O IMPACTO DA COMPOSICÃO SETORIAL, DOS FLUXOS INTRA-SETORIAIS E DA ABERTURA COMERCIAL NA PARTICIPAÇÁO DE MERCADO DAS EXPORTAÇÓES BRASILEIRAS

CLÉSIO LOURENÇO XAVIER E EMERSON FERNANDES MARÇAL

O EFEITO BALASSA-SAMUELSON E A PARIDADE DO PODER DE COMPRA NA ECONOMIA BRASILEIRA CLÁUDIO ROBERTO FÓFFANO VASCONCELOS

CICLOS Y FLUCTUACIONES FINANCIERAS: LA IRREGULAR DINÁMICA ECONÓMICA

SARY LEVY-CARCIENTE

O PENSAMENTO DE KARL POPPER: AS DIFERENTES INTERPRETACÓES DOS METODÓLOCOS DA CIENCIA ECONÓMICA

SOLANCE REGINA MARIN E RAMÓN GARCÍA FERNÁNDEZ

A EXPANSÃO DO ESCOPO TEMÁTICO DAS NEGOCIAÇÓES COLETIVAS DE TRABALHO CARLOS HENRIQUE HORN

EFEITOS DO CAPITAL SOCIAL E DO CAPITAL POLITICO NO DESENVOLVIMENTO ECONOMMICO SIMULAÇOES PARA PAISES E ESTADOS BRASILEIROS

RONALDO A. ARRAES, RICARDO CANDÉA S. BARRETO E VLADIMIR KÜHL TELES

O PROBLEMA DE RISCO MORAL NO MERCADO BRASILEIRO DE ASSISTÊNCIA MÉDICA SUPLEMENTAR LUCIANA PINTO DE ANDRADEE SABINO DA SILVA PÓRTO JÚNIOR

ANO

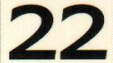

No 41

Março, 2004 
Universidade Federal do Rio Grande do Sul

Reitora: Profo. Wrana Maria Panizzi

Faculdade de Ciencias ECONOMICAS

Diretora: Prof Pedro César Dutra Fonseca

Centro de Estudos e Pesquisas Economicas

Diretor: Prof. Gentil Corazza

Departamento de Ciencias Económicas

Chefe: Prof. Ricardo Dathein

Curso de Pós-Graduação em Economia

Coordenador: Prof. Eduardo Pontual Ribeiro

Programa de Pó-Graduação em Desenvolvimento Rural

Coordenador: Prof. Jalcione Almeida

CONSElHo EDITORIAL:

Carlos G. A. Mielitz Netto (UFRGS), Eduardo A. Maldonado Filho (UFRGS), Eduardo P. Ribeiro (UFRGS), Eleutério F. S. Prado (USP), Eugênio Lagemann (UFRGS), Fernando Cardim de Carvalho (UFRJ), Fernando Ferrari Filho (UFRGS), Fernando de Holanda Barbosa (FGV/RJ), Flávio Vasconcellos Comim (UFRGS), Gentil Corazza (UFRGS), Giácomo Balbinotto Netto (UFRGS), Gustavo Franco (PUC/RJ), Jan A. Kregel (UNCTAD), João Rógério Sanson (UFSC), Joaquim Pinto de Andrade (UnB), Jorge Paulo Araújo (UFRGS), Juan H. Moldau (USP), Marcelo S. Portugal (UFRGS), Maria Alice Lahorgue (UFRGS), Paul Davidson (University of Tennessee), Paulo D. Waquil (UFRGS), Pedro C. D. Fonseca (UFRGS), Philip Arestis (Levy Economics Institut of Bard College), Roberto C. de Moraes (UFRGS), Ronald Otto Hillbrecht (UFRGS), Sabino da Silva Porto Jr. (UFRGS), Stefano Florissi (UFRGS) e Werner Baer (University of Illinois at Urbana-Champaign).

COMISSĀO EDITORIAL:

Eduardo Augusto Maldonado Filho, Fernando Ferrari Filho, Gentil Corazza, Marcelo Savino Portugal, Paulo Dabdab Waquil e Roberto Camps Moraes.

EDITOR: Prof. Fernando Ferrari Filho

Editor Adjunto: Prof. Gentil Corazza

SeCretária: Paulo Roberto Eckert

REVISÃO DE TEXTOS: Vanete Ricacheski

EdITORAÇÃo Eletrónca: Vanessa Hoffmann de Quadros

Fundador: Prof. Antônio Carlos Santos Rosa

Os materiais publicados na revista Análise Econômica são da exclusiva responsabilidade dos autores. É permitida a reprodução total ou parcial dos trabalhos, desde que seja citada a fonte. Aceita-se permuta com revistas congêneres. Aceitam-se, também, livros para divulgação, elaboração de resenhas e recensões. Toda correspondência, material para publicaçāo (vide normas na terceira capa), assinaturas e permutas devem ser dirigidos ao seguinte destinatário:

PROF. FERNANDO FERRARI FILHO Revista Análise Econômica - Av. João Pessoa, 52 CEP 90040-000 PORTO ALEGRE - RS, BRASIL Telefones: (051) 316-3513 - Fax: (051) 316-3990

Análise Econômica E-mail: rae@ufrgs.br

Ano 22, $n^{\circ} 41$, março, 2004 . Porto Alegre

Faculdade de Ciências Econômicas, UFRGS, 2004

Periodicidade semestral, março e setembro.

Tiragem: 500 exemplares

1. Teoria Econômica - Desenvolvimento Regional -

Economia Agrícola - Pesquisa Teórica e Aplicada -

Periódicos. I. Brasil

Faculdade de Ciências Econômicas,

Universidade Federal do Rio Grande do Sul. 


\title{
O Impacto da Composição Setorial, dos Fluxos Intra-Setoriais e da Abertura Comercial na Participação de Mercado das Exportações Brasileiras
}

\author{
Clésio Lourenço Xavier* \\ Emerson Fernandes Marçal ${ }^{* *}$
}

\begin{abstract}
Resumo: O objetivo do trabalho consiste em pesquisar os determinantes da participação de mercado (market share) das exportações brasileiras no mercado internacional no período recente. Com base em dados de painel (panel data), procurar-se-á avaliar o grau de contribuição de alguns fatores na explicação dos market shares brasileiros nos diversos produtos exportados. Três tipos de variáveis serão testados, a saber: a) grau de abertura da economia brasileira; b) importância relativa do produto na pauta de exportação mundial e; c) grau de comércio intra-setorial.
\end{abstract}

Palavras-chave: comércio internacional; comércio intra-setorial; abertura comercial, composição setorial; regressões em painel.

\begin{abstract}
The main goal of this article is to test if Brazilian exports shares in the international market can be explained by the degree of openess of the Brazilian economy; the relative importance of each sector in world exports and the degree of intra-sector trade. It is a panel study and the GMM estimator proposed by Arellano $\mathcal{E}$ Bond is used. The main conclusion is that the selected variables help to explain the evolution of the Brazilian market shares in world trade in the period covered by the sample (1981 to 1997).
\end{abstract}

Key-words: international trade; intra-industry trade; open economy; specialization pattern; panel regressions.

JEL: F14, C23.

\footnotetext{
" Professor Adjunto do IE-UFU - Instituto de Economia da Universidade Federal de UberlândiaMG. E-mail: clesio@ie.ufu.br.

“Doutorando em Economia da USP.E-mail: efmarcal@uol.com.br.
} 


\section{Introdução}

A economia brasileira, após as restrições macroeconômicas do início da década de 1980, foi obrigada a gerar superávits comerciais expressivos para financiar seu balanço de pagamentos. Grande parte de tais superávits foi obtido com limitações tarifárias e não-tarifárias às importaçōes, ou seja, como um processo deliberado de fechamento da economia brasileira. A partir do início dos anos 1990, ocorreu um forte aumento do nível de importações acompanhado de um moderado aumento das exportações.

Até meados da década de 1980 houve um aumento da participação de mercado das exportações brasileiras no mercado mundial. A partir de então, ocorreu uma queda progressiva da participação de mercado brasileira, ou seja, as exportações brasileiras cresceram a um ritmo inferior ao nível mundial e o país perdeu posição relativa.

Esta tendência de queda parece ter sido substituída por um período de estabilidade na participação das exportaçōes no comércio mundial após meados da década de 1990, coincidindo com um período de maior estabilidade monetária.

Além disso, a literatura de comércio exterior, sumariada no próximo item, sugere que a observância da composição setorial das exportações e/ou importações de uma determinada economia visà-vis a composição setorial do comércio mundial - aliada a outros fatores, tais como o grau de abertura e a natureza dos fluxos de comércio setoriais da economia doméstica - é fundamental na compreensão de longo prazo dos saldos comerciais e das restrições externas a que está submetida a economia local. Vale dizer, a existência de padrões de especialização importa na sustentabilidade dos saldos comerciais de um país (CAMUTO, 1998).

De fato, em termos gerais, o que se observou no padrão de especialização das exportações do Brasil no período recente foi uma intensificação da participação de setores com contribuição negativa ao saldo comercial. Em parte, isso ocorreu em função de ausência de competitividade em setores dinâmicos no mercado internacional, inclusive alguns produtos agrícolas e minerais. De outro lado, onde a inserção brasileira é competitiva, uma parcela importante dos setores, produziu impactos negativos sobre o saldo comercial (BAUMANN $\mathcal{E}$ NEVES, 1998). 
No que tange aos saldos comerciais e às restrições externas presentes na economia brasileira ao longo dos últimos vinte anos (1980-1999), ganhos de competitividade "malignos" (por exemplo, taxas de salário nominal/câmbio declinantes) ou "benignos" (aumentos de produtividade, etc.) foram fortemente acionados de modo a compensar os efeitos negativos dos padrões de especialização (CASTRO, 1997 e CASTRO E PIRES de SOUZA, 1985).

O objetivo do trabalho consiste em pesquisar os determinantes da participação de mercado (market share) das exportações brasileiras no mercado internacional no período recente. Com base em dados de painel (panel data), procurar-se-á avaliar o grau de contribuição de alguns fatores na explicação dos market shares brasileiros nos diversos produtos exportados. Três tipos de variáveis serão testados, a saber: a) grau de abertura da economia brasileira; b) importância relativa do produto na pauta de exportação mundial; e c) grau de comércio intra-setorial.

\section{Comércio internacional: especialização e fluxos intra-setoriais}

No modelo ricardiano clássico, pode-se localizar em quais setores os países têm vantagens comparativas a partir das diferenças internacionais de produtividade do trabalho, as quais poderão transformar-se ou não em vantagens absolutas, dependendo das diferenças nas taxas de salários e nas taxas de câmbio. Mas não se pode determinar a composição desses setores dentro dos fluxos comerciais, para tanto se fazendo necessária a determinação da curva de demanda internacional.

Nesta interpretação, os diferenciais internacionais de produtividade constituem-se em um primeiro elemento a ser destacado como determinante da especialização internacional, ainda que não se possa definir sua composição setorial. Na verdade, a especialização ricardiana é sempre uma especialização setorialmente específica (produtos têxteis ou produtos farmacêuticos) determinada pelas vantagens comparativas de acordo com as taxas de crescimento das produtividades setoriais, diferentemente da especialização smithiana, na qual o importante é o grau de especialização determinado pelas economias de escala, desconsiderando-se a especificidade setorial (DOWRICK, 1997). 
Posteriormente, em Dornbusch, Fischer 83 Samuelson (1977), uma das formas de resolução desta indeterminação do modelo ricardiano ocorreu através da utilização de um modelo contínuo de bens, onde se adotou a hipótese de homoteticidade das funções de demanda ${ }^{1}$. Com isso, a parcela da renda gasta em cada um dos bens é constante, mesmo que cresça ou decresça a renda, desaparecendo as diferenças nas elasticidades-renda entre os bens e, por conseguinte, o problema da composição setorial.

No modelo convencional de Heckscher-Ohlin é fundamental a presença das suposições de que a tecnologia é idêntica em todos os países - seja porque é um bem público, ou porque pode ser adquirida a um custo acessível - e de que as curvas de indiferenças são similares entre os parceiros comerciais porque, dada uma mesma inclinação dos termos de troca (preços relativos internacionais), o padrão de especialização dos países ocorrerá naqueles produtos e/ ou setores cuja produção seja mais intensiva no fator de produção localmente abundante, em termos relativos.

Um mecanismo central neste modelo Heckscher-Ohlin de comércio internacional é a existência de flexibilidade intersetoriais rápidas o suficiente para compensar os ajustes intra-setoriais decorrentes da intensificação dos fluxos comerciais. Por isso, economias abertas poderiam obter ganhos líquidos em termos de produtividade e bem-estar em relação à autarquia, sem perda de emprego de qualquer um de seus fatores.

As "novas" teorias do comércio internacional originaram-se a partir de uma revisão parcial do modelo Heckscher-Ohlin - no qual os fluxos comerciais são mais intensos entre os países que possuem diferenças significativas nas dotações de fatores - diante da constatação paradoxal e empírica de que os fluxos comerciais são mais expressivos entre países desenvolvidos, ou seja, economias que possuem dotações de fatores similares (DOSI, PAVITT $\mathcal{Z}$ SOETE, 1990).

Em outros termos, a existência de intensos fluxos comerciais intra-setoriais entre países desenvolvidos representou um fato estilizado suficientemente forte, indicando a necessidade de incor-

\footnotetext{
' Funções que guardam as propriedades das funções homogêneas, entre as quais a de que as taxas marginais de substituição entre os produtos não mudam, diante de variações em seus niveis absolutos, caso mantenham-se os preços relativos.
} 
poração da complementaridade entre as estruturas produtivas dos países na explicação dos respectivos padrões de especialização.

Tal revisão teórica foi feita a partir da incorporação dos supostos da teoria da concorrência imperfeita como base para uma teoria do comércio:

- as economias de escala externas à firma e internas à indústria podem implicar ganhos líquidos na situação de abertura comercial ainda que a preços de equilíbrio mais elevados;

- a diferenciação do produto com livre entrada nos mercados também é incorporada. A abertura comercial permitiria aos consumidores dos países envolvidos se defrontar com uma variedade maior de produtos e as firmas com níveis de demanda superiores em relação à situação de "autarquia".

Essa possibilidade da incorporação da concorrência monopolística preencheu a inadequação dos modelos HeckscherOhlin na explicação dos padrões de especialização dos países, resolvendo o paradoxo, à medida que os fluxos comerciais seriam mais intensos entre países desenvolvidos devido à similaridade de seus padrões de demanda. Isto é, nesses países a produção reagiria à proximidade de seus padrões de demanda com um maior fluxo comercial intra-setorial (Idem).

Note que nesta abordagem de concorrência imperfeita reaparece a indeterminação presente no modelo ricardiano clássico, pois que a diferenciação de produto é incorporada a partir da hipótese de que todos os consumidores preferem uma grande variedade de produtos simétricos (forma funcional de Spence-Dixit-Stiglitz). Entretanto, tal variedade de produtos não é infinita como no modelo contínuo de bens de Dornbusch, Fischer $\mathcal{E}$ Samuelson (1977), porque é limitada pela existência de elevados custos fixos. Ou seja, existem economias de escala associadas aos custos fixos, as quais representam a existência de barreiras à entrada.

De acordo com Krugman (1989, p. 1217-1218), nos modelos de comércio com concorrência imperfeita determina-se apenas o número de bens que é produzido, mas não se pode determinar quais bens serão produzidos devido à hipótese da simetria existente entre os bens, isto é, não se pode explicitar a composição setorial prevalecente. Vale dizer, o padrão de especialização - entendido enquanto composição setorial - novamente é indeterminado em função da presença de economias de escala. 
De outro lado, os fluxos comerciais entre países desenvolvidos e países subdesenvolvidos - o comércio Norte-Sul - continuaria seguindo o modelo clássico Heckscher-Ohlin, através das diferentes dotações de fatores. Dessa forma, o comércio Norte-Sul seria constituído predominantemente de fluxos intersetoriais e não intrasetoriais.

A partir deste brevíssimo survey da literatura, depreende-se a necessidade de verificação empírica da relação existente entre o padrão de especialização de uma determinada economia, vale dizer, a composição setorial de suas exportações em relação ao resto do mundo, bem como a natureza dos fluxos setoriais e o grau de abertura da economia em tela.

Em outros termos, cabe verificar o grau em que a composição setorial das exportaçōes de um país, os fluxos intra-setoriais e a abertura comercial relacionam-se com seus respectivos market shares no comércio internacional. A hipótese a ser testada, no caso da economia brasileira, refere-se à existência de um provável distanciamento da composição setorial da exportações locais em relação à composição setorial das exportações mundiais.

\section{Notas metodológicas}

Os dados relativos aos fluxos de comércio foram obtidos junto às Naçōes Unidas. Os produtos são classificados de acordo com o padrão SITC revisão 2 - Standard International Trade Classification. O nível de desagregação trabalhado é o de três dígitos. Trabalha-se com um total de 229 produtos. O período abrange os anos de 1981 a 1997, e não faltam dados na amostra, vale dizer, trata-se de uma análise de panel balanceada. Os dados coletados para o Brasil são os valores exportados anuais pelo país para o resto do mundo (sem especificação de destino) para todos os produtos. Também dispõese do valor total das exportações realizadas por todos os países do mundo em conjunto. Desta forma, calculou-se a participação de mercado para o produto i dividindo-se o total exportado pelo Brasil do produto i pelo total mundial do mesmo produto. Esta variável é denominada pme.

Calculou-se também a importância relativa do produto i no total mundial. Para isto dividiu-se o total exportado pelos países do 
produto i pelo total mundial de todos os produtos, obtendo assim a composição da pauta de exportações mundial. Esta variável é denominada de $p s w$. A participação no setor externo do produto brasileiro foi obtida a partir da soma de exportação e importação dividido pelo PIB brasileiro em dólares - wt. O total de importação e exportação brasileiro foi coletado junto ao IFS-FMI. Os valores em dólares do PIB brasileiro, no Banco Mundial.

Os dados de comércio intra-setorial foram coletados junto a Terra (1999). Utilizou-se o indicador de Grubel e Loyd que é calculado a partir da seguinte fórmula: $G L_{j}=\frac{\left(X_{j}+M_{j}\right)-\left|X_{j}-M_{j}\right|}{\left(X_{j}+M_{j}\right)}$. Como é possível observar neste trabalho, no período recente ocorreu um aumento do comércio intra-industrial em muitos setores da economia brasileira, permitindo o seguinte comentário de Terra (1999): "Both aggregate indices of intra-industry trade show a steady increase over time for different industries, they increase for most industries. The most spectacular increase is the Textile, Apparel and Footwear industry ${ }^{2} . "$

\section{Modelo Estimado}

O principal objetivo do trabalho consiste avaliar em que medida as variáveis propostas contribuem para explicar a evolução da participaçāo de mercado das exportações brasileiras. Nesta perspectiva, em primeiro lugar, a inclusão da variável participação do produto no total exportado mundial (psw) como variável explicativa numa regressão para a participaçāo de mercado tenta avaliar em que medida o comportamento da primeira variável está condicionada pela evolução da participação do setor no mundo. A existência desta relação seria evidência de que o país estaria acompanhando um padrão de comércio mundial, ou seja, o país estaria aumentando participação de mercado em produtos de setores que estão ampliando sua participação no comércio mundial.

Em segundo lugar, a inclusão da variável participação do setor externo no produto interno bruto brasileiro busca avaliar se o grau

\footnotetext{
¿ É um fato reportado na literatura que o índice de Grubel $\mathcal{E}$ Loyd sofre forte oscilação conforme o nivel de agregação utilizado. Quanto mais agregados forem os dados, maiores são os valores assumidos pelo indicador. Muito do comércio intra-setorial reportado para niveis maiores de agregação caem sensivelmente quando dados com alto nível de desagregação são analisados. Qual nível de agregação utilizar em trabalhos empíricos é um ponto em aberto na literatura. Ver sobre este ponto Deardoff (1984, p. 501-502).
} 
de abertura da economia condicionou positiva ou negativamente a participação de mercado dos diversos produtos exportados pelo Brasil. Deseja-se averiguar se os momentos em que houve um aumento do tamanho do setor externo na economia brasileira coincidiram com os momentos em houve aumento generalizado da participação de mercado das exportações brasileiras nos diversos produtos e a magnitude de tal efeito.

Calcula-se o índice de abertura a partir da média das exportações e importaçōes em relação ao PIB, ou seja, [(X+M)/2]/PIB. Em meados da década de 1980 houve uma queda da participação do setor externo no PIB brasileiro. Este período coincide com a queda da participação de mercado do Brasil no comércio mundial, indicando a existência de uma possível relação entre grau de abertura e participação de mercado das exportações brasileiras.

A última variável incluída como explicativa é uma medida do grau de comércio intra-setorial com o objetivo de averiguar se uma alteração do padrão de comércio intra-setorial tem alguma relação com a participação de mercado do país nos diversos produtos dos setores. Em princípio, um setor em que o país apresenta grande vantagem comparativa deve ocorrer um elevado saldo comercial setorial em relação ao total exportado e um baixo nível de importação. Neste caso, o índice de Grubel $\mathcal{E} 3$ Loyd deveria apresentar valores baixos. Por outro lado, tal índice também pode estar sendo influenciado pela abertura econômica. Um forte aumento do nível exportado e importado com uma deterioração do saldo comercial faria com que o índice de Grubel e Loyd aumentasse.

Dessa forma, estima-se se existe alguma relação entre as variáveis listadas e participação de mercado do país ${ }^{3}$ :

$$
p m e=f(w t, g l, p s w)
$$

Os sinais esperados para as relações sugeridas acima são:

$$
\frac{\partial p m e}{\partial w t}=(+) ; \frac{\partial p m e}{\partial g l}=(+/-) \text { e; } \frac{\partial p m e}{\partial p s w} \geq 0 \text {. }
$$

3 Outras variáveis poderiam ser incluídas na regressão, tais como diferenças nas relações capital produto dos diversos setores, índices de produtividade, indicadores sobre as características tecnológicas dos diversos produtos produzidos. Sobre este último ponto pode-se citar o trabalho de Lodwinger (1975). O objetivo do autor é analisar os determinantes da participação de mercado das exportaçōes das indústrias americanas no mundo. Como variáveis explicativas, o autor utiliza indicadores do grau de intensidade tecnológica dos produtos, entre outros fatores. Entretanto pelo fato destes, para o Brasil, serem de difícil obtenção e ou construção, utilizaramse as variáveis já mencionadas. Uma resenha sobre trabalhos empíricos na área de comércio internacional pode ser encontrada em Deardorff (1984). 


\section{Procedimentos Econométricos}

\subsection{Modelos Sem Dinâmica}

Um possível modelo a ser estimado consiste em:

$\mathrm{Y}_{\mathrm{it}}=\mathrm{f}_{\mathrm{i}}+\beta \mathrm{X}_{\mathrm{it}}+\mathrm{u}_{\mathrm{it}}(\mathrm{i})$

em que $f_{i}$ é um componente fixo ligado à unidade $i ; X_{i t}$ representa o conjunto de vetores com as variáveis explicativas; $Y_{i 1}$, a variável dependente; e $\mathrm{u}_{\mathrm{it}}$, choques aleatórios normais e independentes ao longo do tempo.

O objetivo consiste em obter uma estimador consistente de b e com propriedades desejadas de eficiência. A escolha da técnica de estimação a ser utilizada depende das hipóteses assumidas quanto à relação existente entre o erro aleatório $\left(\mathrm{u}_{\mathrm{i} i}\right)$ e os regressores $\left(\mathrm{X}_{\mathrm{it}}\right)$; quanto ao erro aleatório e o efeito fixo $\left(f_{i}\right)$.

No caso mais restritivo, pode-se assumir que $\mathrm{E}\left(\mathrm{f}_{\mathrm{i}}, \mathrm{X}_{\mathrm{it}}\right)=0-$ ortogonalidade entre o efeito fixo e os regressores - e $E\left(u_{i t}, X_{i t-s}\right)=0$ para qualquer s. Pode-se utilizar OLS (Mínimos Quadrados Ordinários) ou LSDV (Mínimos Quadrados com Dummies para cada unidade). Ambos os estimadores são consistentes sendo que o segundo é mais eficiente.

Abrindo mão da hipótese de ortogonalidade do efeito fixo e dos regressores, ou seja, assumindo que $E\left(\mathrm{f}_{\mathrm{i}}, \mathrm{X}_{\mathrm{i}}\right) \neq 0$, não é possível mais assumir consistência para OLS, contudo LSDV continua sendo consistente. Um outro estimador consistente possível é OLS utilizando as variáveis em primeira diferença (FD-OLS) ${ }^{4}$. Este estimador também é consistente para o caso proposto no parágrafo anterior, mas apresenta problemas de eficiência.

Pode-se também assumir que $\mathrm{E}\left(\mathrm{f}_{\mathrm{i}}, \mathrm{X}_{\mathrm{it}}\right)=0$ e $\mathrm{E}\left(\mathrm{u}_{\mathrm{it}}, \mathrm{X}_{\mathrm{it}}\right) \neq 0$. Neste caso, nenhum dos estimadores propostos acima (OLS, LSDV ou FD-OLS) são consistentes. Para obter estimativas consistentes de b é necessário utilizar Variáveis Instrumentais ou GMM (Método dos Momentos Generalizados).

Em vez de ser propor (1) como ponto de partida, pode-se optar por:

$$
\mathrm{Y}_{\mathrm{it}}=\beta \mathrm{X}_{\mathrm{it}}+\mathrm{e}_{\mathrm{i}}+\mathrm{u}_{\mathrm{it}}
$$

\footnotetext{
${ }^{4}$ Tomando a primeira diferença de (1), o efeito fixo é eliminado.
} 
A diferença básica entre as duas formas está na maneira como é tratada a diferença existente entre as diversas unidades. No primeiro caso, as unidades são diferentes por conta de algum fator determinista constante ao longo do tempo, enquanto, no segundo caso, as diferenças surgem por conta de algum fator aleatório $\left(e_{i}\right)$ que atingiu cada unidade de forma diferenciada. A estimaçāo de (2) pode ser feita por GLS. Note que é necessário postular $E\left(u_{i t}, X_{i t}\right)=0$ para qualquer s para ter-se consistência dos parâmetros.

\subsection{Modelos Com Dinâmica}

Nos modelos (1) e (2) não há variáveis defasadas, seja regressores ou a variável dependente. Em vez do modelo (1) ou (2) pode-se propor o modelo (3) apresentado abaixo:

$$
\mathrm{Y}_{\mathrm{it}}=\mathrm{f}_{\mathrm{i}}+\rho \mathrm{Y}_{\mathrm{it}-1}+\beta_{1} \mathrm{X}_{\mathrm{it}}+\beta_{2} \mathrm{X}_{\mathrm{it}-1}+\mathrm{u}_{\mathrm{it}} \text { (3) }
$$

Assumindo que $\mathrm{E}\left(\mathrm{u}_{\mathrm{it}}, \mathrm{X}_{\mathrm{it} \cdot \mathrm{s}}\right)=\mathrm{E}\left(\mathrm{f}_{\mathrm{i}}, \mathrm{X}_{\mathrm{it}-\mathrm{s}}\right)=0$ para $\mathrm{s}=0,1$ os parâmetros $\beta_{1}$ e $\beta_{2}$ podem ser estimados de forma consistente por qualquer dos métodos sugeridos acima. Contudo, não é possível obter uma estimativa consistente do parâmetro $\rho$. É necessário utilizar algum instrumento para obter uma estimativa consistente de $\rho$. As variáveis $\Delta Y_{t-j}$ e $Y_{i t-j}$ são candidatas naturais a instrumento ${ }^{5}$, pois $E\left[\left(\Delta Y_{i t}-\rho \Delta Y_{i t-1}-\beta_{1} \Delta X_{i t}-\beta_{2} \Delta X_{i t-1}\right) Y_{i t \cdot j}\right]=E\left[\left(\Delta Y_{i t}-\rho \Delta Y_{i t-1}-\beta_{1} \Delta X_{i t}-\beta_{2} \Delta X_{i t-1}\right) \Delta Y_{i t i t}\right]=0$ para $(\mathrm{j}=2, \ldots, \mathrm{t}-1 ; \mathrm{t}=2, \ldots ., \mathrm{T})$. Caso $\mathrm{E}\left(\mathrm{X}_{\mathrm{it}-\mathrm{s}} \mathrm{u}_{\mathrm{i}}\right) \neq 0$ e $\mathrm{E}\left(\mathrm{f}_{\mathrm{i}}, \mathrm{X}_{\mathrm{it}}\right) \neq 0$ para $s=0,1$, OLS e LSDV não fornecem estimativas consistentes de $\beta_{1}$ e $\beta_{2}$. É necessário utilizar os regressores em primeira diferença e instrumentalizar $\Delta \mathrm{X}_{\mathrm{t}} \mathrm{e} \Delta \mathrm{X}_{\mathrm{t}-1}$, utilizando, por exemplo $\mathrm{X}_{\mathrm{t} t 2}$ ou $\Delta \mathrm{X}_{\mathrm{t}-2}$. Este estimador é proposto por Hsiao e Anderson (AD).

Outra abordagem consiste em utilizar GMM (Método dos Momentos Generalizados) na forma proposta por Arellano $\mathcal{E}$ Bond (1990). A partir de (3) é possivel chegar a:

$$
\Delta \mathrm{Y}_{\mathrm{it}}=\rho \Delta \mathrm{Y}_{\mathrm{it}-1}+\beta_{1} \Delta \mathrm{X}_{\mathrm{it}}+\beta_{2} \Delta \mathrm{X}_{\mathrm{tit}-1}+\mathrm{u}_{\mathrm{it}}-\mathrm{u}_{\mathrm{it}-1}
$$

Existem duas diferenças básicas entre (3) e (4): a) O efeito fixo, $f_{i}$, presente em (3) foi eliminado de (4) pela diferenciação; e b) introduziu-se autocorrelação de primeira ordem em (4). Embora o estimador HD permita obter estimativas consistentes de todos os parâmetros,

\footnotetext{
${ }^{5}$ Este estimadores foram propostos por Anderson e Hsiao (HD). Ver Arellano $\mathcal{E}$ Bond (1990, p.278).
} 
não possui as propriedades desejadas de eficiência. A primeira perda de eficiência advém da autocorrelação automática nos erros que não é levada em conta pelo estimador. A possível presença de heterocedasticidade também adicionaria perda adicional de eficiência.

Arellano E3 Bond (1990) propõem o seguinte procedimento em dois estágios para estimar a equação (4), apresentado abaixo.

Supondo que $\mathrm{E}\left(\mathrm{u}_{\mathrm{it}}\right)=0, \mathrm{E}\left(\mathrm{u}_{\mathrm{it}}{ }^{2}\right)=\sigma^{2}$ e $\mathrm{E}\left(\mathrm{u}_{\mathrm{it}} \mathrm{u}_{\mathrm{it}-\mathrm{s}}\right)=0$ para qualquer $\mathrm{s}$, a matriz de variância e covariâncias dos erros em (4) é dada por:

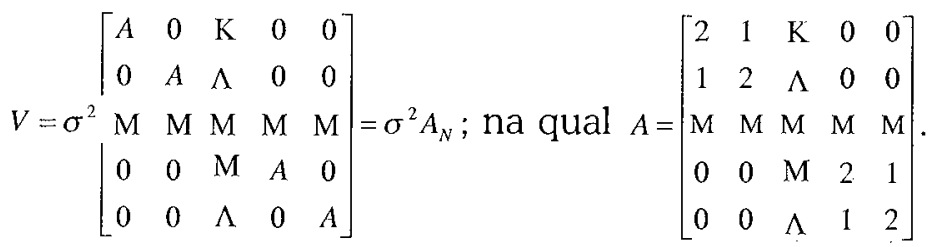

O primeiro estágio consiste em obter uma estimativa consistente de $\beta=\left[\rho \beta_{1} \beta_{2}\right]$ através de:

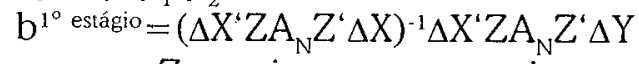

em que $Z$ consiste numa matriz com um conjunto de instrumentos. A matriz para o caso em que todos os regressores são estritamente exógenos é dada por $Z=\left[\operatorname{diag}\left(y_{1} \wedge y_{T-s}\right) \vdots\left(\Delta x_{s+1} \Lambda \Delta x_{T}\right)\right]$ e para o caso em um grupo de regressores é estritamente exógeno $\left(\mathrm{x}_{1}\right)$ e outro predeterminado $\left(\mathrm{x}_{2}\right)$ é dado por:

$$
Z=\left\lfloor\operatorname{diag}\left(y_{1} \wedge y_{T-s} x_{1}^{1} \wedge x_{T-s}^{1}\right) \vdots\left(\Delta x_{s+}^{2} \wedge \Delta x_{T}^{2}\right)^{\prime}\right\rfloor \text { para } \mathrm{s}=2, \Lambda ., \mathrm{T}-2 .
$$

$O$ segundo estágio consiste obter uma estimativa utilizando a matriz de $\mathrm{V}$ de variâncias e covariâncias construídas a partir dos resíduos estimados no primeiro estágio:

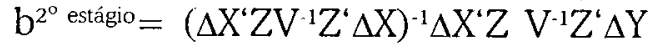

Existem três tipos de estimativas de desvios padrões dos estimadores. A primeira consiste na estimativa tradicional a partir dos resultados do primeiro estágio. A segunda estimativa consiste em construir a matriz de variância e covariância a partir da metodologia proposta por Hansen (1982). A terceira consiste em utilizar a matriz de variância e covariância estimada a partir dos resultados do segundo estágio. Os estimadores serão denominados, respectivamente, de primeiro estágio, robusto e de segundo estágio. Todos os três são equivalentes assintoticamente na ausência de 
heterocedasticidade e correlação cruzada dos erros. Na presença de um destes problemas, os dois últimos estimadores são preferíveis. Arellano E3 Bond (1990) recomendam a realização de inferência estatística a partir da estimativa robusta, pois acreditam que deva existir uma tendência a subestimação dos desvios padrões a partir do estimador em dois estágios em pequenas amostras.

\subsection{Testes de Especificação}

Arellano $\mathcal{E}$ Bond (1990) propõem três testes de especificação para avaliar se a equação (3) é um modelo adequado ou não. O primeiro deles consiste em testar se existe evidência de autocorrelação nos resíduos estimados a partir das estimativas de $1^{\circ}$ e $2^{\circ}$ estágios dos parâmetros. A evidência de autocorrelação invalidaria a especificação dado que a consistência dos estimadores é afetada. O segundo teste consiste num teste de superidentificaçāo proposto por Sargan (1958, 1988). Com base neste teste, verifica-se se um conjunto de instrumentos utilizados são ortogonais aos resíduos estimados.

O terceiro teste é um teste de Hausman ${ }^{6}$ sobre o coeficiente da variável dependente defasada e pode ser implementado de forma seqüencial. Caso a primeira defasagem $\left(\mathrm{Y}_{\mathrm{it}-\mathrm{l}}\right)$ não seja um instrumento válido, haverá correlação entre a variável e os erros, e logo as estimativas obtidas a partir de um GMM que utilize apenas esta condição de momento serão inconsistentes, enquanto um GMM que utilize todas as outras condições de momentos fornecerá estimativas consistentes. Isto é o necessário para um teste de Hausman. Caso seja rejeitada a hipótese nula, isto é evidência de autocorrelação de primeira ordem, dado que o coeficiente obtido está viesado. A rigor este teste de Hausman pode ser aplicado em qualquer situação em que se deseja comparar uma estimativa consistente tanto sob hipótese nula ou alternativa com um estimador apenas consistente sobre a alternativa. Uma variante do teste de Sargan é obtida a partir da

${ }^{6}$ A estatística de Hausman pode ser calculada a partir de: $\left[\begin{array}{c}b_{1}-\beta_{1} \\ b_{2}-\beta_{2} \\ \vdots \\ b_{k}-\beta_{k}\end{array}\right][\operatorname{Var}(b)-\operatorname{Var}(\beta)]^{-1}\left[\begin{array}{c}b_{1}-\beta_{1} \\ b_{2}-\beta_{2} \\ \vdots \\ b_{k}-\beta_{K}\end{array}\right]-\chi^{2}(K) \mathrm{em}$ que b é um conjunto de estimativas de parâmetros obtidas a partir de um estimador consistente apenas sob a hipótese nula, enquanto b é consistente sob a nula é a alternativa. 
comparação da diferença entre as estatísticas de Sargan para superidentificação nos dois casos acima. Sob a hipótese nula, esta estatística tende a ser zero. $\mathrm{Na}$ alternativa, tal diferença deve ser positiva. A estatística converge para um distribuição qui-quadrado.

\section{Resultados}

O objetivo desta seção consiste em apresentar os resultados obtidos a partir da estimação de diversos modelos para explicar a evolução da participação de mercado brasileira. Na primeira seção, apresentam-se os modelos em que não há dinâmica. Já na segunda, apresentam-se os modelos em que há dinâmica presente. Por fim, um balanço dos resultados obtidos é feito.

Modelos de Painel sem Dinâmica:

Estimou-se a seguinte equação:

$\mathrm{pme}_{\mathrm{it}}=\mathrm{f}_{\mathrm{i}}+\beta_{\mathrm{o}} \mathrm{wt} \mathrm{t}_{\mathrm{t}}+\gamma_{0} \mathrm{psw}_{\mathrm{it}}+\phi_{0} \mathrm{gl}_{\mathrm{jt}}+\mathrm{u}_{\mathrm{it}}$

Os resultados estão apresentados na Tabela 1 .

Tabela 1: Resultados das Estimativas da Equação (1) por OLS, LSDV e RE

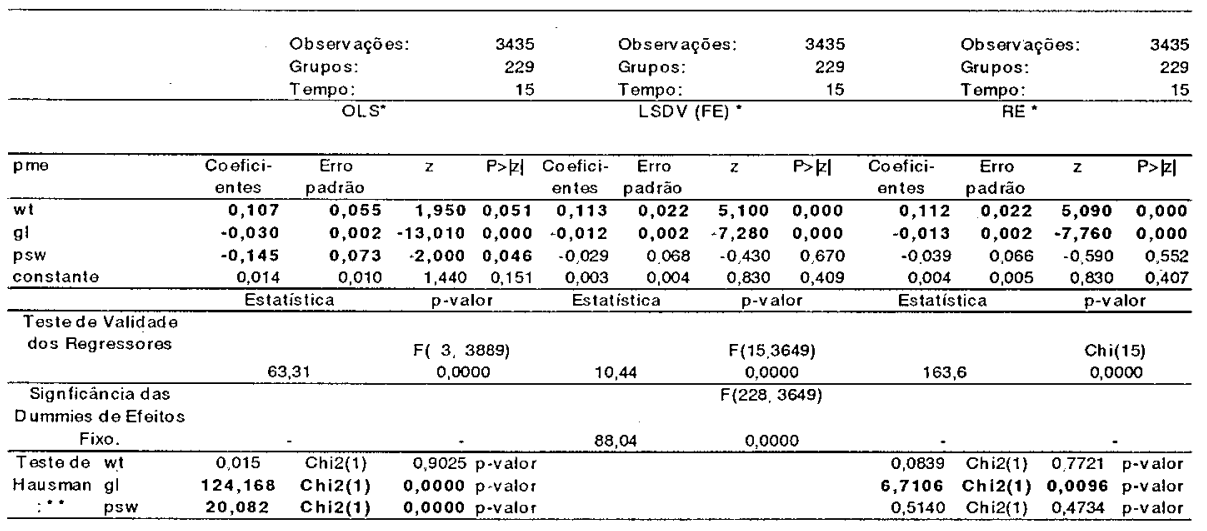

- Contém Dummies de ano.

* Primeira coluna - Comparação de EF e EA; segunda coluna - Comparação de OLS com EF.

As estimativas dos parâmetros obtidas por OLS diferem fortemente das obtidas para os modelos de efeito Fixo e Aleatório indicando que $\mathrm{E}\left(\mathrm{f}_{\mathrm{i}} \mathrm{X}_{\mathrm{it}}\right) \neq 0$. Neste caso as estimativas obtidas por OLS estão viesadas. Os parâmetros associados às variáveis wt e gl são significa- 
tivos em todos os modelos, enquanto a variável psw é significativa apenas para o caso OLS.

Realizou-se o teste de Hausman para avaliar qual modelo é preferível: Efeito Fixo (FE) e Efeito Aleatório (RE). Sob a hipótese nula, tanto o modelo de $\mathrm{FE}$ quanto de RE são consistentes, sendo que o segundo é mais eficiente. Sob a hipótese alternativa, o modelo RE fornece estimativa inconsistente dos parâmetros e, desta forma, a diferença entre os dois estimadores deve ser diferente de zero. Raciocínio semelhante vale para comparações entre OLS e LSDV.

Como pode ser observado na Tabela 1, assumir ortogonalidade dos regressores e efeitos fixos não deve ser uma hipótese válida. $\mathrm{O}$ único regressor para o qual a estatística de Hausman não é significativa é o grau de abertura (wt), algo que parece ser intuitivo em princípio. É pouco provável que algo específico a um mercado de um produto específico condicione a evolução de uma variável agregada como grau de abertura.

Modelos de Painel com Dinâmica:

Duas equações foram estimadas e são representadas abaixo: $\mathrm{pme}_{\mathrm{it}}=\mathrm{f}_{\mathrm{i}}+\rho_{1} \mathrm{pme}_{\mathrm{it}-1}+\beta_{0} \mathrm{w}_{\mathrm{t}}+\beta_{1} \mathrm{w}_{\mathrm{t}-\mathrm{I}}+\gamma_{0} \mathrm{psw}_{\mathrm{it}}+\gamma_{1} \mathrm{psw}_{\mathrm{it}-1}+\phi_{0} \mathrm{gl}_{\mathrm{jt}}+\phi_{1} \mathrm{gl}_{\mathrm{jt}-\mathrm{f}}$ ${ }_{1}+u_{i t}$

na qual pme representa a participação das exportações do produto i do país no total mundial do setor i; psw representa a participação do produto i no comércio internacional total; e w representa a participação do comércio exterior $(X+M)$ no produto brasileiro.

$\mathrm{pme}_{\mathrm{it}}=\mathrm{f}_{\mathrm{i}}+\mathrm{r}_{\mathrm{i}} \mathrm{pme}_{\mathrm{it}-1}+\mathrm{b}_{0} \mathrm{w}_{\mathrm{t}}+\mathrm{b}_{1} \mathrm{w}_{\mathrm{t}-1}+\mathrm{g}_{00 \mathrm{o}} \log (\mathrm{psw})_{\mathrm{it}}+\mathrm{g}_{1} \log (\mathrm{psw})_{\mathrm{it}-\mathrm{f}}$ ${ }_{1}+f_{0} g l_{j t}+f_{1} g l_{j t-1}+u_{i t}$

na qual $\log (\mathrm{psw})_{\mathrm{it}}$ representa o logaritmo da participação do produto i no total exportado no mundo deste produto.

A segunda especificação permite dar maior flexibilidade ao modelo na medida em que os efeitos marginais da variável $\mathrm{psw}_{\mathrm{it}}$ na

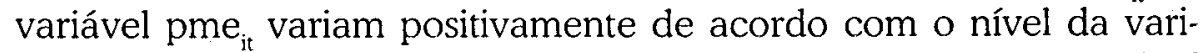
ável $\mathrm{psw}_{\mathrm{it}}$. Em setores que a variável $\mathrm{psw}_{\mathrm{it}}$ é maior, o efeito marginal sobre a participação de mercado é maior. A justificativa para esta especificação é que a evolução do produto na pauta de exportação mundial é um importante condicionante para a evolução da participação de mercado nos diversos produtos, mas esta importância é menor em setores mais importantes do comércio mundial

Na equação (2), o efeito marginal de longo prazo é dado por: $\frac{\partial p m e_{i}}{\partial p s w_{L P}}=\frac{\gamma_{1}+\gamma_{2}}{1-\rho_{1}}$. Já na equação 3 , o efeito muda para 
$\left(\frac{\partial p m e_{i}}{\partial p s w_{i}}\right)_{L P}=\frac{\gamma_{1}+\gamma_{2}}{1-\rho_{1}} \frac{1}{\left(p s w_{i}\right)_{L P}}$ - por conta da introdução da transformação logarítmica das variáveis explicativas - ou seja, a magnitude do efeito marginal passa a depender inversamente do nível da variável. No caso de valores extremos para a variável explicativa $p s w, o$ efeito destas sobre a variável explicada cai. A razão desta especificação consiste em tentar introduzir algum tipo de não linearidade na análise para a variável em questão.

Tabela 2: Estimação da equação (2) utilizando GMM

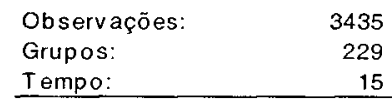

Modelo 1

Resultados de primeiro Estágio: Resultados de primeiro Estágio:

Regressōes Estritamente Exógenos Regressões Predeterminados - (wt gl - (wt g|psw). psw)

\begin{tabular}{|c|c|c|c|c|c|c|c|c|c|}
\hline pme & & $\begin{array}{l}\text { Coefici- } \\
\text { entes }\end{array}$ & \multirow[t]{2}{*}{$\begin{array}{c}\text { Erro } \\
\text { padrāo } \\
\text { robust } \\
0 \\
0,086\end{array}$} & $z$ & $P>|z|$ & $\begin{array}{l}\text { Coefici- } \\
\text { entes }\end{array}$ & $\begin{array}{c}\text { Erro } \\
\text { padrão } \\
\text { robusto }\end{array}$ & $z$ & $P>|z|$ \\
\hline pme & LD & 0,378 & & 4,400 & 0,000 & 0,391 & 0,087 & 4,490 & $0, \overline{000}$ \\
\hline \multirow[t]{2}{*}{$w t$} & D 1 & 0,224 & 0,076 & 2,930 & 0,003 & 0,229 & 0,076 & 2,990 & 0,003 \\
\hline & LD & 0,160 & 0,064 & 2,490 & 0,013 & 0,161 & 0,065 & 2,500 & 0,013 \\
\hline \multirow[t]{2}{*}{ gl } & D1 & $.0,015$ & 0,004 & $-3,410$ & 0,001 & $-0,012$ & 0,003 & $-4,060$ & 0,000 \\
\hline & LD & - & - & - & - & 0,005 & 0,002 & 3,140 & 0,002 \\
\hline \multirow[t]{2}{*}{ psw } & D 1 & - & - & - & - & - & . & - & $\cdot$ \\
\hline & LD & - & - & - & - & - & - & - & - \\
\hline \multirow{2}{*}{\multicolumn{2}{|c|}{ constante }} & $-0,003$ & 0,001 & $-2,120$ & 0,034 & $-0,003$ & 0,001 & $-2,140$ & 0,032 \\
\hline & & \multicolumn{2}{|c|}{ Estatistica } & \multicolumn{2}{|c|}{$\mathrm{p}-\mathrm{v}$ alor } & \multicolumn{2}{|c|}{ Estatistica } & \multicolumn{2}{|c|}{ p-valor } \\
\hline \multicolumn{10}{|c|}{ HO: A usência de } \\
\hline \multicolumn{2}{|c|}{ A utocorrelaçāo nos } & $-2,22$ & & 0,027 & & $-2,23$ & & 0,026 & \\
\hline \multicolumn{2}{|c|}{$\begin{array}{l}\text { HO: A usência de } \\
\text { A utocorrelação nos }\end{array}$} & $-0,71$ & & 0,4787 & & $-0,67$ & & 0,5006 & \\
\hline \multicolumn{2}{|c|}{$\begin{array}{l}\text { Teste de Validade } \\
\text { dos Regressores - }\end{array}$} & 234,97 & $\operatorname{chi2}(16$ & \multicolumn{2}{|c|}{0,0000} & 231,32 & $\operatorname{chi2}(17)$ & \multicolumn{2}{|c|}{0,0000} \\
\hline \multicolumn{2}{|c|}{ Teste de } & 210,00 & $\operatorname{chi} 2(11$ & \multicolumn{2}{|c|}{0,0000} & 227,44 & $\operatorname{chi} 2(247)$ & \multicolumn{2}{|c|}{0,8090} \\
\hline
\end{tabular}

* Teste de Autocorrelação realizado a partir das estimativas de segundo estágio.

** Teste de Sargan realizado a partir das estimativas de segundo estágio.

(1) Em todos os modelos foram incluidas dummies de ano.

Os resultados obtidos na estimação do modelo da equação (2) são reportados na Tabela 2. Numa especificação, obtiveram-se as estimativas supondo que os regressores sejam estritamente exógenos. Desta forma, apenas a variável dependente defasada foi instrumentalizada. Os resultados dos testes de especificação não se 
mostraram adequados. A hipótese nula no teste de Sargan é rejeita$\mathrm{da}$, indicando que algum dos instrumentos pode não ser adequado. A estatística para avaliar a presença de autocorrelação de primeira ordem nos resíduos está na fronteira. A cinco porcento, rejeita-se a hipótese nula. Já a 1\%, aceita-se a hipótese nula. Uma possível explicação seria a presença de endogenidade, ou seja, assumir exogenidade estrita dos regressores seria a causa dos problemas verificados nos testes de especificação.

Tabela 3: Estimação da equação (3) utilizando GMM

\begin{tabular}{|c|c|c|c|c|c|c|c|c|c|}
\hline \multicolumn{2}{|c|}{$\begin{array}{l}\text { Observaçōes: } \\
\text { Grupos: } \\
\text { Tempo: }\end{array}$} & $\begin{array}{r}3435 \\
229 \\
15 \\
\end{array}$ & & & & & & & \\
\hline & & \multicolumn{8}{|c|}{ Modelo 2 (1) } \\
\hline & & \multicolumn{4}{|c|}{$\begin{array}{c}\text { Resultados de primeiro Estágio: } \\
\text { Regressões Estritamente Exógenos (wt, } \\
\text { gl, } \log (p s w) \text { ). }\end{array}$} & \multicolumn{4}{|c|}{$\begin{array}{c}\text { Resultados de primeiro Estágio: } \\
\text { Regressōes Predeterminados - (wt, } \\
\text { gl, } \log (p s w))\end{array}$} \\
\hline pme & & $\begin{array}{l}\text { Coefici- } \\
\text { entes }\end{array}$ & $\begin{array}{c}\text { Erro } \\
\text { padrão } \\
\text { robusto }\end{array}$ & $\bar{z}$ & $\mathrm{P}>|\mathrm{z}|$ & $\begin{array}{l}\text { Coefici- } \\
\text { entes }\end{array}$ & $\begin{array}{c}\text { Erro } \\
\text { padräo } \\
\text { robusto }\end{array}$ & $\bar{z}$ & $P>|z|$ \\
\hline prne & LD & 0,368 & $0, \overline{091}$ & 4,050 & 0,000 & 0,385 & 0,091 & 4,240 & 0,000 \\
\hline \multirow[t]{2}{*}{ wt } & D1 & 0,221 & 0,077 & 2,880 & 0,004 & 0,227 & 0,077 & 2,960 & 0,003 \\
\hline & L.D & 0,155 & 0,064 & 2,420 & 0,015 & 0,157 & 0,064 & 2,430 & 0,015 \\
\hline \multirow[t]{2}{*}{ gl } & DI & $-0,013$ & 0,004 & $-3,400$ & 0,001 & $-0,008$ & 0,003 & $-2,860$ & 0,004 \\
\hline & L.D & - & - & - & 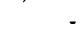 & 0,006 & 0,002 & 3,380 & 0,001 \\
\hline \multirow[t]{2}{*}{$\log (p s w)$} & DI & - & - & - & - & $\cdot$ & - & - & \\
\hline & L.D & 0,017 & 0,003 & 5,170 & 0,000 & 0,015 & 0,004 & 3,710 & 0,000 \\
\hline \multirow[t]{2}{*}{ constante } & & $-0,003$ & 0,001 & $-2,120$ & 0,034 & $-0,003$ & 0,001 & $-2,140$ & 0,032 \\
\hline & 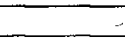 & Estatíst & tica & p-valor & & Estati & Ística & $p-v$ & \\
\hline \multicolumn{10}{|c|}{ H0: Ausência de } \\
\hline \multicolumn{2}{|c|}{$\begin{array}{l}\text { Autocor relação nos } \\
\text { resíduos de primeira } \\
\text { ordem * }\end{array}$} & $-2,17$ & & 0,0304 & & $-2,18$ & & & \\
\hline \multicolumn{2}{|c|}{$\begin{array}{l}\text { H0: Ausência de } \\
\text { Autocorrelação nos } \\
\text { resíduos de segunda } \\
\text { ordem. }\end{array}$} & $-0,85$ & & 0,3938 & & $-0,80$ & & & \\
\hline \multicolumn{2}{|c|}{$\begin{array}{l}\text { Teste de Validade dos } \\
\text { Regressores - chi2(k) - }\end{array}$} & 178,12 & chi2(17) & 0,0000 & & 169,1 & $\operatorname{chi} 2(18)$ & & \\
\hline \multicolumn{2}{|c|}{$\begin{array}{l}\text { Teste de } \\
\text { superidentificação de } \\
\text { chi } 2(19)\end{array}$} & 199,82 & $\operatorname{chi2(119)}$ & 0,0000 & & 226,47 & $\operatorname{chi} 2(311)$ & & \\
\hline
\end{tabular}

* Teste de Autocorrelação realizado a partir das estimativas de segundo estágio.

* Teste de Sargan realizado a partir das estimativas de segundo estágio.

(1) Em todos os modelos foram incluídas dummies de ano.

(2) Utilizaram-se como instrumentos até 5 defasagens de wt, gl, log(psw) e a primeira e segunda de Vantagem Comparativa Revelada (Balassa, 1977).

Os testes de especificação melhoraram quando foi assumido que os regressores são predeterminados em vez de estritamente 
exógenos. Apenas as variáveis comércio intra-setorial (gl) e grau de abertura (wt) são significativas.

Por outro lado, a não-significância da importância do produto na pauta mundial (psw) para explicar a participação de mercado motivou a estimação do modelo da equação (3), reportados na Tabela 3.

No modelo da equação 3, novamente os resultados dos testes de especificação para a equação com regressores estritamente exógenos não são bons. Já quando à estimação, é realizada sob a hipótese de que as variáveis são predeterminadas, os resultados dos testes de especificação são melhores, embora ainda a estatística para detectar autocorrelação de primeira ordem nos resíduos esteja na fronteira entre $5 \%$ e $1 \%$ com p-valor de 0,0289 .

Os resultados são similares aos obtidos na Tabela 2 no que tange às variáveis de comércio intra-setorial (gl) e grau de abertura (wt). A diferença surge com relação á variável pauta mundial quando inserida em logaritmo. Esta variável defasada é significativa, mostrando que a evolução da pauta mundial é importante condicionante da evolução da partiçipação de mercado dos produtos brasileiros no exterior, embora seus efeitos sobre a participação de mercado demorem um período para serem sentidos.

\section{Considerações Finais}

Todos os resultados obtidos são esperados em termos de magnitude e sinal. A partir disso, três conclusões de maior vulto podem ser extraídas a partir dos resultados apresentados anteriormente:

- Em primeiro lugar, o grau de abertura da economia brasileira condicionou de forma positiva o comportamento das participações de mercado, ou seja, em momentos em que o setor externo ganhou importância relativa, houve um ganho generalizado da participação de mercado nos diversos produtos;

- Em todos os modelos analisados, os fluxos de comércio intrasetoriais apresentaram resultados significativos, demonstrando a relação existente entre elevada participação de mercado e baixo índice de comércio intra-setorial;

- Em terceiro lugar, a principal conclusão a ressaltar é o efeito da pauta mundial sobre a participação de mercado das exportaçōes brasileiras: este efeito apresentou-se positivo, ou seja, o país ganhou 
participação de mercado em produtos cuja participação no total mundial vem aumentando; por outro lado, este efeito torna-se menor, quanto maior é a participação relativa do produto na pauta mundial.

Portanto, no período analisado, as exportações brasileiras vêm ampliando sua participação de mercado em setores dinâmicos do comércio mundial, embora ainda persista um relativo distanciamento da pauta de exportação brasileira em relação à pauta de exportação do resto do mundo, isto é, o padrão de especialização local ainda apresenta uma composição setorial distinta da composição setorial prevalecente no comércio mundial.

\section{Referências Bibliográficas}

ANDERSON, T.W. e HSIAO, C. (1982); "Formulation and Estimation of Dynamic Models using Panel Data". In: Journal of Econometrics, 18, 47-82.

ARELLANO, M. e BOND, S. (1991); "Some Tests of Specification for Panel Data: Monte Carlo Evidence and an application to employment equations". In: The Review of Economic Studies, v. 58(2), nº 194, April.

BALASSA, B. (1977); "Revealed Comparative Advantage Revisited: An analysis of relative export shares of industrial countries, 1953-71"; The Manchester School, vol. XLV, nº 4, pp. 327-344.

Baumann, R. $E$ Neves, L. F. C. (1998). "Abertura, Barreiras Comerciais Externase Desempenho Exportador Brasileiro". CEPAL - Brasil, LC/BRS/DT.016".

CANUTO, O. (1998), "Padrões de Especialização, hiatos tecnológicos e crescimento com restrição de divisas". Revista de Economia Política, v. 18, n. $^{\circ} 3$ (71), jul.-set.

CASTRO, A. B. (1997). “O Plano Real e o reposicionamento das empresas.". In: Velloso, J. P. R. (org.), Brasil: desafios de um país em transformação. José Olympio, Rio de Janeiro.

$\mathcal{E}$ Pires de Souza, F. E.(1985). A Economia brasileira em marcha forçada. São Paulo, Paz e Terra.

DEARDOFF, Alan, V. (1984); "Testing Trade Theories and Predicting Trade Flows"; In: Jones, R. W. e Kenen, P. B.; Handbook of International Economics, v. I, capítulo 10, Elsivier Publishers.

DORNBUSCH, R., FISCHER, S. ESAMUELSON, P. (1977). "Comparative advantage trade and payments in a ricardian model with a continuum of goods". In: American Economic Review;v. 67, p. 823-839. 
DOSI, G., PAVITT, K. ESOETE, L. (1990). The economics of technological change and international trade Brighton, Wheatshaf.

DOWRICK, S. (1997). "Innovation and Growth: implications of the New Theory and Evidence." Em: J.Fagerberg, L. Lundberg, P. Hansson and A. Melchior (orgs.), Technology and international trade, Edward Elgar, Cheltenham.

GREENE, W. H. (1999). Econometric Analysis, Fourth edition, Prentice Hall.

HANSEN, L. P. (1982) "Large Sample Properties of Generalized Methods of Moments"; Econometrica, 50, 1029-1054.

KRUGMAN, P. (1989). "Industrial organization and international trade", In: Handbook of industrial organization, v. ii, Schmalensee, R. E Willig, R. (orgs.), Elsevier Science Publishers, North-Holland.

LOWINGER, T. C. (1975); "The techonology factor and export performance of U.S. manufacturing industry"; Economic Inquiry, 13 (junho); 221-236.

TERRA, M. C. (1999). "Finance and Changing Trade Patterns in Brazil"; Textos de Seminários Acadêmicos nº 08/99, Universidade de São Paulo, São Paulo. 